\title{
Comparison of 2D and 3D Local Binary Pattern in Lung Cancer Diagnosis
}

\author{
Kohei Arai \\ Graduate School of Science and \\ Engineering \\ Saga University \\ Saga City, Japan
}

\author{
Yeni Herdiyeni \\ Department of Computer Science \\ Bogor Agricultural University \\ West Java, Indonesia
}

\author{
Hiroshi Okumura \\ Graduate School of Science and \\ Engineering \\ Saga University \\ Saga City, Japan
}

\begin{abstract}
Comparative study between 2D and 3D Local Binary Patter (LBP) methods for extraction from Computed Tomography (CT) imagery data in lung cancer diagnosis is conducted. The lung image classification is performed using probabilistic neural network (PNN) with histogram similarity as distance measure. The technique is evaluated on a set of CT lung images from Japan Society of Computer Aided Diagnosis of Medical Images. Experimental results show that 3D LBP has superior performance in accuracy compare to 2D LBP. The 2D LBP and 3D LBP achieved a classification accuracy of $43 \%$ and $\mathbf{7 8 \%}$ respectively.
\end{abstract}

Keywords- lung cancer detection; local binary pattern; probabilistic neural network.

\section{INTRODUCTION}

Lung cancer is one of the main cause deaths in the world among both men and woman, with an impressive rate about five million deadly cases per year [1]. Lung cancer is a disease of abnormal cells multiplying and growing into a tumor. Cancer cell can be carried away from the lungs in the blood, or lymph fluid that surrounds lung tissue. Lymph flows through lymphatic vessels, which drain into lymph nodes located in the lungs and in the center of the chest. Lung cancer often spreads toward the center of the chest because the natural flow of lymph out of the lungs is toward the center of the chest.

There are two major groups of lung cancer, Small Cell Lung Cancer (SCLC) and Non-Small Cell Lung Cancer (NSCLC). SCLC is a disease in which malignant (cancer) cells form in the tissues of the lung. Also known as oat cell cancer, NSCLC is the most common form of lung cancer that incorporates a variety of cancer sub types such as Squamous cell carcinoma, adenocarcinoma, and large cell carcinoma. This type of cancer also spreads to other parts of the body but tends to progress slowly to different parts of the body slower than SCLC.

One of the main imaging modality for this kind of disease diagnostic is computerized tomography $(\mathrm{CT})^{1}$ of the patient chest.

The advantage of the X-ray CT is pulmonary nodules that are typical shadow of pathological changes of the lung cancer can be detected more clearly compared to the chest X-ray examination even if they are at early stages. A number of methods have been explored for lung cancer detection using CT images. [2,3,4] uses measures on co-occurrence matrices, measures on run-length matrices, moments of the attenuation or intensity histogram, and in some cases fractal dimension as features. Sluimer et al. [5] used a filter bank of Gaussians and Gaussian derivatives.

The system using standard local binary patterns also has been developed and tested to 2D lung CT images. Sorensen et al [6] used joint local binary patterns (LBP) and intensity histogram for discriminating different texture pattern in 2D lung CT images. The extension of original LBP from 2D images to $3 \mathrm{D}$ volume data has been applied to facial expression [7].

[8] has been compared 3D texture representation for 3D brain MR images. The experimental results have shown that 3D LBP perform best with precision recall of $65 \%$ compare to 3D Grey Level Co-occurrence Matrices (3D GLCM), 3D Wavelet Transform (3D WT) [9], [10] and 3D Gabor Transform (3D GT).

In this research, we compare 2D LBP [11] and 3D LBP [7] as a texture features for lung cancer detection. We use probabilistic neural network (PNN) classifier [12] with histogram similarity as distance measure. To the best of our knowledge, this is the first application to use 3D LBP on lung CT image.

\section{PROPOSED METHOD}

\section{A. Framework}

In the following section the framework system is describe. The system is divided into two stages: training and test. In the training-stage, well-segmented lung are loaded into a feature extraction module.

In this research, we used $2 \mathrm{D} \mathrm{LBP}$ and $3 \mathrm{D}$ LBP to extract texture feature of segmented lung. Based on the extracted feature vector from LBP, the trainer classifier will classify the lung cancer image. We used probabilistic neural network (PNN) as features classifier. The system flow is illustrated in Figure 1.

\footnotetext{
${ }^{1}$ http://en.wikipedia.org/wiki/X-ray_computed_tomography
} 


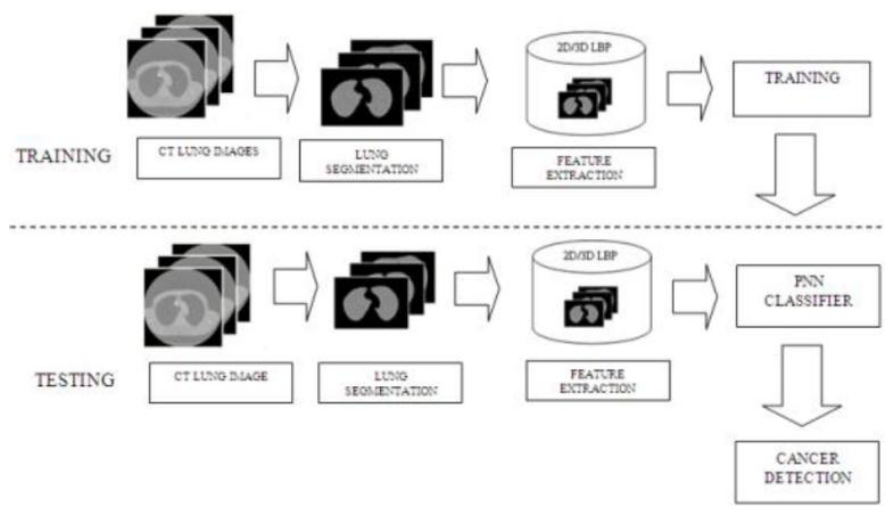

Figure 1. The framework system

\section{B. Lung Segmentation}

This section describes segmentation of the lung cancer. The goal of segmentation is to simplify the representation of an image into something that is more meaningful and easier to analyze.

In medical imaging, segmentation is important for feature extraction, image measurement and image display. In this research lung segmentation is done to eliminate of all artifacts external to patient's body and removal of thorax remaining just parenchyma. We used image enhancement and morphological operation for lung segmentation in the CT image to achieve a better orientation in the image.

Left and right lung are segmented using morphological operation and adaptive threshold based on statistical criteria. Morphology is a technique of image processing based on shape. The value of each pixel in the output image is based on comparison of the corresponding pixel in the input image with its neighbors.

\section{2D Local Binary Pattern (2DLBP)}

After lung segmentation, the next step is feature extraction. Feature extraction is the process of defining a set of features, or image characteristic, which will most efficiently or meaningfully represent information that is important for analysis and classification. For 2D LBP, the features extraction that we use based on the local binary patterns (LBP) proposed by Ojala et al. [9].

In general, LBP measures the local structures at a given pixel using $P$ samples on a circle of radius $R$ around the pixel and summarizes this information with a unique code for each local structure or pattern (Figure 2).

The operator is highly non-linear and detects microstructures in the image at different resolutions governed by the parameter $R$, for example spots, edges, corners, etc., exemplified in the right part of Figure 3.

To obtain LBP value, thresholding performed on the neighborhood circular pixels using the central pixel, and then multiply by binary weighting. As an example for the sampling points $P=8$ and radius $R=1$, the calculation of LBP value is illustrated in Figure 4.

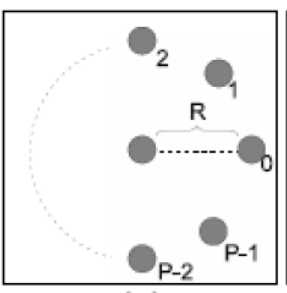

(a)

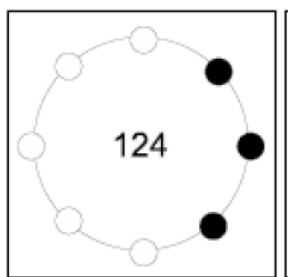

(c)

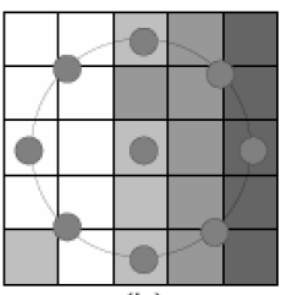

(b)

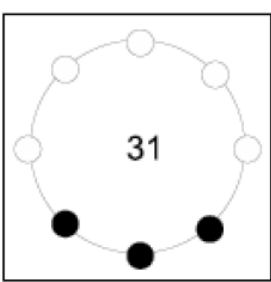

(d)
Figure 2. Illustration of LBP. (a) The filter is defined by two parameters; the circle radius $R$ and the number of samples $P$ on the circle. (b) Local structure is measured with reference to a given pixel by placing the center of the circle in the position of that pixel. (c) The samples on the circle are binarized by thresholding with the intensity in the center pixel as threshold value. Black is zero and white is one. (d) Rotating the example image in (b) ninety degrees clock wise reduce the LBP code to 31 which is smallest possible code for this binary pattern. This principle is used to achieve rotation invariant.

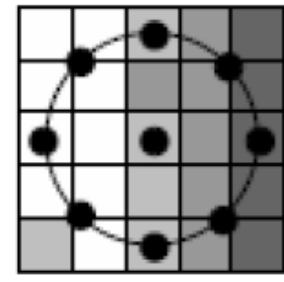

(a)

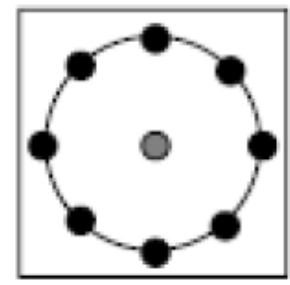

Spot

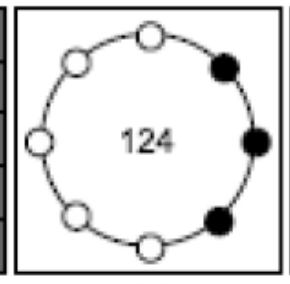

(b)

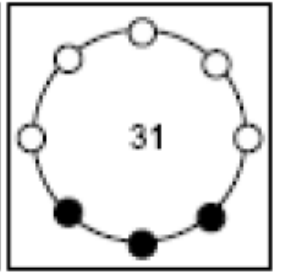

(c)
Figure 3. Some of the microstructures that LBP are measuring

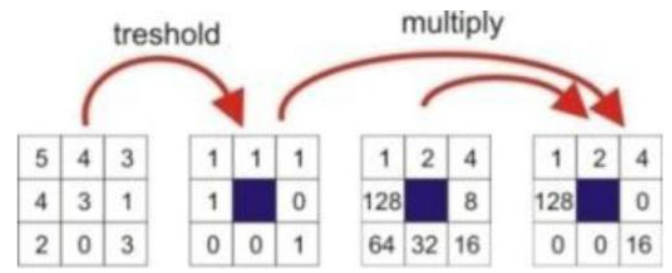

Figure 4. Calculation of LBP value.

LBP can be formulated as follows:

$L B P_{P, R}\left(x_{c}, y_{c}\right)=\sum_{p=0}^{P-1} s\left(g_{p}-g_{c}\right) 2^{p}$ 


$$
s(x)= \begin{cases}1 & x \geq 0 \\ 0 & x<0\end{cases}
$$

where $x_{c}$ and $y_{c}$ are the coordinate of center pixel, $p$ is circular sampling points, $P$ is number of sampling points or neighborhood pixels, $g_{p}$ is gray scale value of $p, g_{c}$ is center pixel, and $s$ or sign is threshold function. For classification purpose, the LBP values are represented as a histogram.

\section{3D Local Binary Pattern (3DLBP)}

3D local binary pattern (3D LBP) or volume local binary pattern (VLBP) is an extension of LBP operator that combining the motion and appearance [7]. The features extracted in a small local neighborhood of the volume are not insensitive with respect to translation and rotation, but also robust with respect to monotonic gray-scale changes. In terms of 3D LBP form, Zhao, et. al [7] have proposed a 3D dynamic texture recognition by concatenating three histogram obtained from LBP on three orthogonal planes (LBP-TOP), i.e., XY, $\mathrm{XZ}$ and $\mathrm{Y}$ planes, the idea that has been adopted in this investigation.

VLBP is defined as the joint distribution $V$ of gray level of $3 P+3(P>1)$ image pixel [7]. $P$ is the number of local neighboring points around the central pixel in one frame. Both are shown in equation (3).

$$
\begin{aligned}
& V=v\left(g_{t_{c}-L, c}, g_{t_{c}-L, 0}, \ldots . . g_{t_{c}-L, P-1}, g_{t_{c}, 0}, \ldots,\right. \\
& \left.g_{t_{c}, P-1}, g_{t_{c}+L, 0}, \ldots . g_{t_{c}+L, P-1}, g_{t_{c}+L, c}\right) .
\end{aligned}
$$

where the gray value $g_{t_{c}, c}$ corresponds to the gray value of the center pixel of the local volume neighborhood, $g_{t_{c}-L, c}$ and

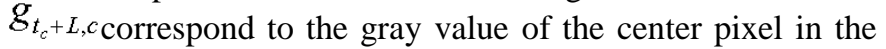
previous and posterior neighboring frames with time interval $L ; g_{t, p}\left(t=t_{c}-L, t_{c}, t_{c}+L ; p=0, \ldots, P-1\right)$ correspond to the gray values of $P$ equally spaced pixel on a circle of radius $R$ $(R>0)$ in image $t$, which form a circularly symmetric neighbor set.

Volume local binary pattern $\left(V L B P_{L, P, R}\right)$ characterizes the spatial structure of the local volume dynamic textures shown in equation (4).

$V L B P_{L, P, R}=\sum_{q=0}^{3 P+1} v_{q} 2^{q}$

Figure 6 shows the whole computing procedures for $V L B P_{1,4,1}$. We begin by sampling neighboring points in the volume, and then thresholding every point in the neighborhood with the value of the center pixel to get a binary value. Finally we produce the VLBP code by multiplying the threshold binary values with weights given to the corresponding pixel and sum up the results.

The basic VLBP code is calculated for each pixel in the cropped portion of the DT, and the distribution of the code is used as a feature vector denoted by $\mathrm{D}$ shown in equation (5):

$$
\begin{aligned}
& D=v\left(U L B P_{L, P, R}(x, y, t)\right), x \in\{\lceil R\rceil, \ldots, X-1-\lceil R\rceil\}, \\
& y \in\{[R\rceil, \ldots, Y-1-\lceil R]\}, t \in\{\lceil L\rceil, \ldots, T-1-\lceil L]
\end{aligned}
$$

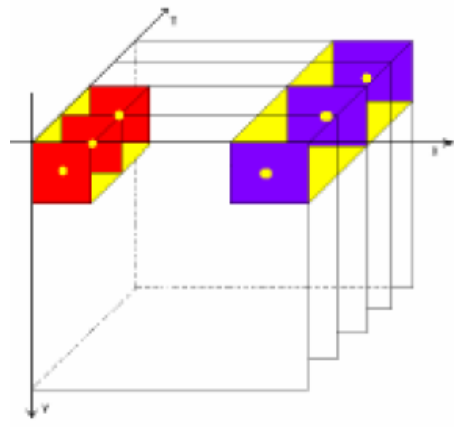

(a)

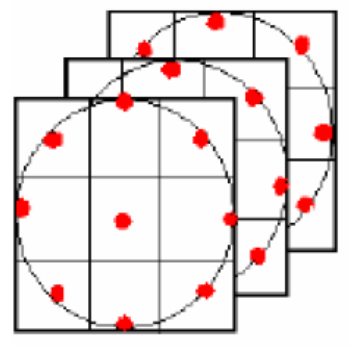

(b)

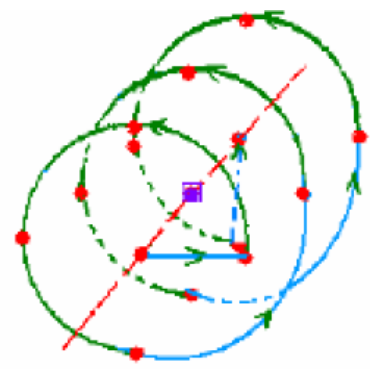

(c)
Figure 5. (a) Volume in dynamic texture (Right volume with $\mathrm{L}=1$, while left volume with $\mathrm{L}=2$. (b) Circularly symmetric neighbor sets in volume $(\mathrm{R}=1$ and $\mathrm{P}=8)$, (c) Neighboring points along the helix surface of the cylinder $(\mathrm{P}=4)$.

The histograms are normalized with respect to volume size variations by setting the sum of their bins to unity. Because the dynamic texture is viewed as sets of volumes and their features are extracted on the basis of those volume textons ${ }^{2}$, VLBP combines the motion and appearance to describe dynamic texture [7].

To make the VLBP computationally simple and easy to extend, only the co-occurrences on three separated planes are then considered. The textures are modeled with concatenated Local Binary Pattern histograms from Three Orthogonal Planes (LBP-TOP). The circular neighborhoods are generalized to elliptical sampling to fit the space-time statistics. A block-based approach combining pixel-level, region-level and volume-level features is proposed for dealing with such nontraditional dynamic textures in which local information and its spatial locations should also be taken into account.

The LBP-TOP (LBP on three orthogonal planes) only considers the co-occurrences statistics in three direction, $\mathrm{XY}$, $\mathrm{XT}$, and YT. LBP-TOP code is extracted from the XY, XT and YT planes, which are denoted as XY-LBP, XT-LBP, and YT-LBP, for all pixels and statistic of three different planes are obtained, and then concatenated into a single histogram. The procedure is demonstrated in Figure 7.

There are two differences between VLBP and LBP-TOP. Firstly, the VLBP uses three parallel planes of which only the middle one contains the center pixel. The LBP-TOP, on the other hand, uses three orthogonal planes which intersect in the center pixel.

\footnotetext{
${ }^{2}$ http://www.cis.upenn.edu/ jshi/papers/ICCV99b_final.pdf
} 


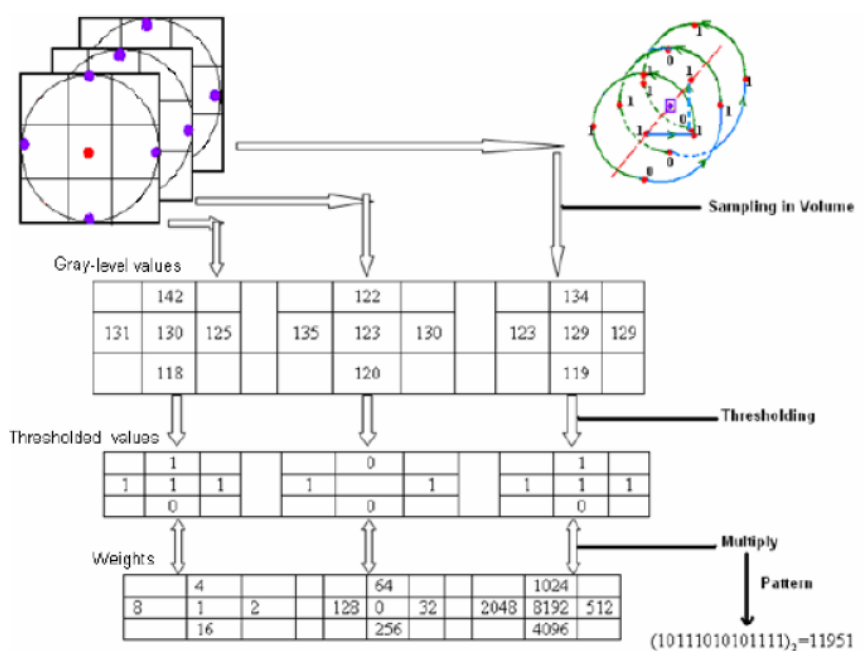

Figure 6. Procedure of VLBP1,4,1

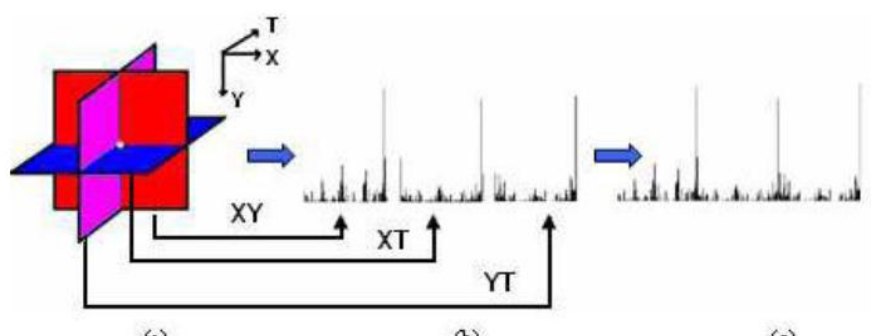

(a)

(b)

(c)

Figure 7. (a) Three planes in 3D-LBP (b) LBP histogram from each plane (c) Concatenated feature histogram

Secondly, VLBP considers the co-occurrences of all neighboring points from three parallel frames, which tends to make the feature vector too long. LBP-TOP considers the features distributions from each separate plane and then concatenates them together, making the feature vector much shorter when the number of neighboring points increase.

\section{E. Probabilistic Neural Network(PNN)}

Probabilistic Neural Network (PNN) proposed by Donald Specht in 1990 as an alternative back-propagation neural network. PNN has several advantages i.e. training requires only one iteration, and general solution is obtained by using a Bayesian approach [13]. PNN is a neural network that uses radial basis function (RBF). RBF is a function that is shaped like a bell that scales a nonlinear variable [14].

PNN consists of four layers, input layer, pattern layer, summation layer and output layer. PNN structure is shown in Figure 8. The layers that make up the PNN are as:

\section{1) Input layer}

Input layer is input $x$ consisting of $k$ value to be classified in one class of $n$ classes.

\section{2) Pattern layer}

Pattern layer performs dot product between input and weight $x_{i j}$, or $Z_{i}=x \cdot x_{i j}$, then divided by a certain bias $\sigma$ then inserted into the radial basis functions, that is $\operatorname{radbas}(n)=\exp (-n)^{2}$.
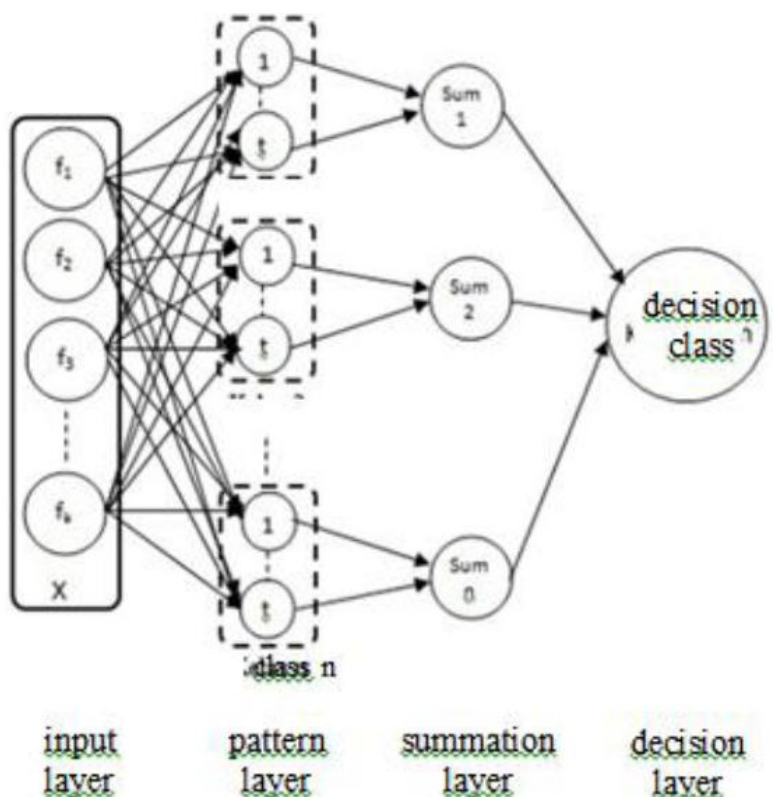

Figure 8 Structure of PNN.

Thus, the equation used in pattern layer is computed as follows:

$$
f(x)=\exp \left(-\frac{\left(x-x_{i j}\right)^{T}\left(x-x_{i j}\right)}{2 \sigma^{2}}\right)^{2}
$$

with $x_{i j}$ express training vector class $i$ order $j$.

\section{3) Summation layer}

In this layer, each pattern in each class is summed to produce a population density function for each class. The equation used at this layer is as follows:

$p(x)=\frac{1}{(2 \pi)^{\frac{k}{2}} \sigma^{k} t} \sum_{i=1}^{t} \exp \left(-\frac{\left(x-x_{i j}\right)^{T}\left(x-x_{i j}\right)}{2 \sigma^{2}}\right)^{2}$

4) Output layer

At the decision layer input $x$ will be classified into class $I$ if the value is larger than any other class.

\section{F. Cross Validation}

Cross-validation is widely used for model assessment and comparison. Cross-validation is a method of measuring generalization error through the use of holdout data. In this research we used $K$-fold cross validation. In this procedure, dataset $D$ is divided into $K$ partitions of roughly equal size, $D=\bigcup_{k=1}^{k} D_{k}$

$k=1$ and each partition is termed a "fold" of the dataset (thus there are $K$-folds). The model is trained on $K-1$ folds and the $\mathrm{K}$-th fold is used for testing [10]. This repeated $\mathrm{K}$ times such that each fold is used for testing exactly once.

The $K$-fold cross validation procedure is often applied to choose a model specific parameter. Suppose that the models are indexed by parameter $\lambda \in \Lambda$ with corresponding 
estimated model function $f^{-k(i)}$ to be evaluated on the $k$-th fold. The optimal $\hat{\lambda}$ is chosen as follows:

$\lambda=\underset{\lambda}{\arg \min } K C V(\lambda)=\underset{\lambda}{\arg \min } \frac{1}{n} \sum_{i=1}^{n}\left(y_{i}-y_{\lambda}{ }^{-k(i)}\right)^{2}$

The final model is trained with the optimal parameter over the entire data, with the KCV statistic reported as the cross validation prediction error.

\section{G. Performance Measures}

To evaluate performance of the system, we used receiver operating characteristic curve (ROC) ${ }^{3}$ analysis. Four basic measures are defined from the set of true conditions and observed information as shown in Figure 9.

These basic measures are true positive, false positive, false negative and true negative rates or fraction. A "positive" observation in an image means that the object was observed in the test. A "negative" observation means that the object was not observed in the test. A "true condition" is the actual truth, while an observation is the outcome of the test.

A graph between TP and FP is called receiver operating characteristic (ROC) curve for a specific medical imaging or diagnostic test for detection of an object. A true positive fraction is also called the sensitivity while the true negative fraction is known as specificity of the test for detection of an object. In each test, we measured the following values:

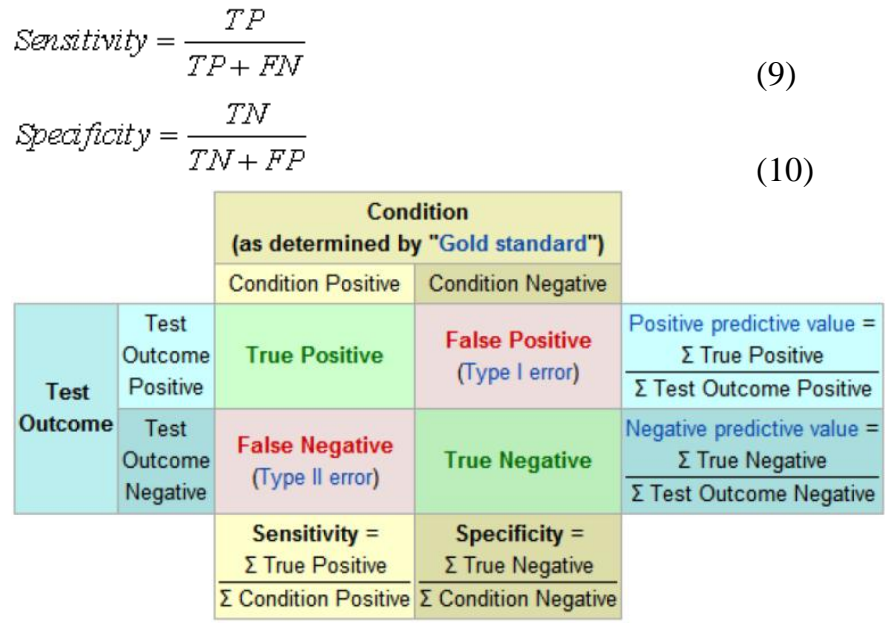

Figure 9. A conditional matrix for defining four basic performance measures of receiver operating characteristics curve (ROC) analysis.

- True positive fraction (TP) is the ratio of the number of positive observations to the number of positive true condition cases.

- False negative fraction $(\mathrm{FN})$ is the ratio of the number of negative observations to the number of positive true condition cases.

- False positive fraction (FP) is the ratio of the number of positive observations to the number of negative true condition cases.

- True negative fraction (TN) is the ratio of the number of negative observations to the number of negative true condition cases.

Accuracy of the test is given by a ratio of correct

\footnotetext{
${ }^{3}$ http://en.wikipedia.org/wiki/Receiver_operating_characteristic
}

observation to the total number of examination cases. Thus, the accuracy is expressed with equation (11).

$$
\text { Accuracy }=\frac{T P+T N}{T P+T N+F P+F N}
$$

\section{EXPERIMENTAL RESULTS}

\section{A. Data Collections}

The input of our system is a set of lung CT images with doses from $150 \mathrm{mAs}$ to $200 \mathrm{mAs}$, in plane resolution $0.63 \mathrm{~mm} / \mathrm{pixel}$, slice thickness of $5 \mathrm{~mm}$. The number of data is 14 patients' data. The image sizes are all 512 by 512 pixel within cross section, with 43 to 81 slices in $\mathrm{Z}$ direction. Data set are collected from Japan Society of Computer Aided Diagnosis of Medical Images. There are 14 patients and five classes of lung cancer that we used in the experiments:

1) Small cell carcinoma (6 patients)

2) Tubercoluma of the lung (2 patients)

3) Inflammantory pseudotumor (2 patients)

4) Adecarcinoma (1 patient)

5) Squamous cell carcinoma (3 patients)

Due to the fact that we only have a relatively smaller size of database, then we divide data into two types cancer, benign (non-cancerous) and malignant (cancerous). So, in these experiments, tubercoluma of the lung and inflammantory pseudotumor we classify into benign and the remaining data we classify into malignant. Before feature extraction stage, lung CT images are segmented. The process is started with thorax extraction. This stage comprises the removal of all artifacts external to the patient's body. There are mainly consists of five steps for lung segmentation, i.e.:

1) Crop the original image from 512 by 512 pixels to 466 by 351 pixels to get the lung region.

2) Compute the original image histogram. Apply adaptive threshold based on statistical criteria using Otsu Algorithm ${ }^{4}$ from original image histogram to make binary image.

3) Remove object which touching the border.

4) Apply morphology operation by apply Structuring Element (SE) to an input image. In this step we use erosion and dilation to remove pixel on the object boundaries. The number of pixel removed from the object in an image depends on the size of structuring element used to process the image. In this research we used SE (3 by 3 ).

Process flow is illustrated in Figure 10. After lung segmentation, the next stage is feature extraction. In our study, we use original LBP (2D LBP) [9] and volume LBP (3D LBP) [7] to extract lung cancer features. There three experiments we conducted to compare performance in accuracy of 2D LBP and 3D LBP.

In this study, the 3D LBP-TOP uses three orthogonal planes that intersect in the center voxel ${ }^{5}$ as shown in Figure 11.

\footnotetext{
${ }^{4}$ http://en.wikipedia.org/wiki/Otsu's_method

${ }^{5}$ http://www.webopedia.com/TERM/V/voxel.html
} 


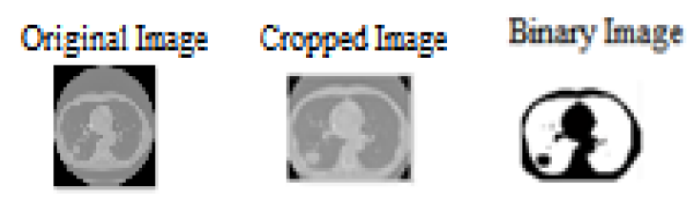

Border Cleared Lung Mask Lung Extracted

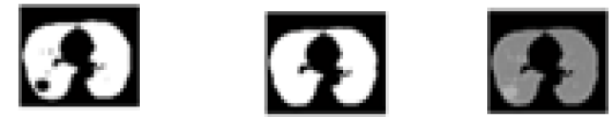

Figure 10. Lung segmentation

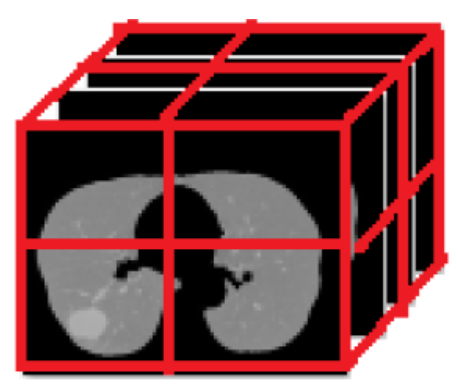

Figure 11. Block for 3D lung cancer.

In the experiment we select 4 and 8 neighbors with 1 voxel radius as a local neighborhood. Fifty-nine uniform LBP code is then extracted from XY, XT, YT planes respectively, producing a 59 bin histogram for each plane by accumulating the 59 binary patterns. Finally, three histogram are concatenated to generate a 3D texture representation, giving the size of a feature vector being 177 (=59 by 3 ).

In order to describe local features parts of a lung, in this experiment 3D volume is divided into four non-overlapping equality sized blocks, which are 2 block along each of $x, y, z$ axes respectively as shown in Figure 8. The method of 3D LBP is applied to extract local features from each block respectively. Therefore, the dimension of the feature vector for 3D lung cancer is the size of local features multiplied by 4 .

\section{B. Results}

In this section, the performance of 2D LBP and 3D LBP in lung cancer detection is evaluated. In the experiment we select 8 neighbors with 1 voxel radius as a local neighborhood. Table 1 presents the overall classification rates of 2D LBP and Table 4 presents the overall classification rates of 3D LBP. Table 2,3 shows ROC analyzed results for original $2 \mathrm{D}$ LBR and 2D block LBR, respectively. Meanwhile, Table 5,6 shows ROC analyzed results for original 3D LBR and 3D block LBR, respectively.

As shown in Table 1 and Table 4, in 2D LBP and 3D LBP, the block LBP method has better recognition rates compare to original LBP. Experimental results show that $3 \mathrm{D}$ block LBP is superior to 2D block LBP in accuracy. The 2D Block LBP and 3D block LBP achieved a classification accuracy of $43 \%$ and $78 \%$ respectively. We only use a relatively small size of database in the experiments so further study will be needed to verify this finding.
TABLE I. THE CLASSIFICATION RATES OF 2D LBR $(8,1)$

\begin{tabular}{|c|r|r|}
\hline Sample & $\begin{array}{l}\text { Accuracy } \\
\text { (Original) }\end{array}$ & $\begin{array}{l}\text { Accuracy } \\
\text { (Block) }\end{array}$ \\
\hline 1 & 0.30 & 0.41 \\
\hline 2 & 0.38 & 0.42 \\
\hline 3 & 0.43 & 0.50 \\
\hline 4 & 0.39 & 0.43 \\
\hline 5 & 0.34 & 0.41 \\
\hline 6 & 0.29 & 0.44 \\
\hline 7 & 0.36 & 0.42 \\
\hline Average & 0.36 & 0.43 \\
\hline \multicolumn{2}{|c|}{ Note : (Neighbor points, Radius) }
\end{tabular}

TABLE II. ROC ANANALYSIS FOR ORIGINAL 2D LBR $(8,1)$

\begin{tabular}{|l|c|c|}
\hline $0=$ benign & $0=$ benign & $1=$ malignant \\
\hline $1=$ malignant & 3 & 6 \\
\hline & $\begin{array}{c}\text { Sensitivity } \\
=0\end{array}$ & $\begin{array}{c}\text { Specificity } \\
=0.46\end{array}$ \\
\hline
\end{tabular}

TABLE III. ROC ANALYSIS FOR 2D BLOCK LBR $(8,1)$

\begin{tabular}{|l|c|c|}
\hline & $0=$ benign & $1=$ malignant \\
\hline $0=$ benign & 0 & 5 \\
\hline $1=$ malignant & 3 & 6 \\
\hline & $\begin{array}{c}\text { Sensitivity } \\
=0\end{array}$ & $\begin{array}{c}\text { Specificity } \\
=0.54\end{array}$ \\
\hline
\end{tabular}

TABLE IV. The ClassificAtion RATES OF 3 D LBP $(8,8,8,1,1,1,1)$

\begin{tabular}{|c|r|r|}
\hline Sample & $\begin{array}{r}\text { Accuracy } \\
\text { (Original) }\end{array}$ & $\begin{array}{l}\text { Accuracy } \\
\text { (Block) }\end{array}$ \\
\hline 1 & 0.54 & 1.00 \\
\hline 2 & 0.75 & 0.75 \\
\hline 3 & 0.68 & 0.75 \\
\hline 4 & 0.75 & 0.73 \\
\hline 5 & 0.78 & 0.73 \\
\hline 6 & 0.73 & 0.69 \\
\hline 7 & 0.72 & 0.78 \\
\hline Average & 0.71 & 0.78 \\
\hline
\end{tabular}

Note : (Neighbor point (XY, XT, YT), FxRadius, FyRadius, Interval) 
TABLE V. ROC ANALYSIS OF ORIGINAL 3D LBP

\begin{tabular}{|l|c|c|}
\hline & $0=$ benign & $1=$ malignant \\
\hline $0=$ benign & 0 & 0 \\
\hline $1=$ malignant & 4 & 10 \\
\hline & $\begin{array}{c}\text { Sensitivity } \\
=0\end{array}$ & $\begin{array}{c}\text { Specificity } \\
=1\end{array}$ \\
\hline
\end{tabular}

TABLE VI. ROC ANALYSIS OF 3D BLOCK LBP

\begin{tabular}{|l|c|c|}
\hline & $0=$ benign & $1=$ malignant \\
\hline $0=$ benign & 1 & 0 \\
\hline $1=$ malignant & 3 & 10 \\
\hline & $\begin{array}{c}\text { Sensitivity } \\
=0.25\end{array}$ & $\begin{array}{c}\text { Specificity } \\
=1\end{array}$ \\
\hline
\end{tabular}

\section{CONCLUSION AND FUtURE DiRECTION}

We conducted a comparative study between two techniques of feature extraction from lung computed tomography images for lung cancer diagnosis. According to experiments results, the block LBP method has better recognition rates compared to the original LBP.

As overall, it may conclude that 3D LBP is superior to 2D LBP in accuracy.

The 2D block LBP and 3D block LBP achieved a classification accuracy of $43 \%$ and $78 \%$, respectively. Due to the fact that we only have a relatively smaller size of database, further study is needed to justify these results.

\section{ACKNOWLEDGMENT}

This work is financially funded by Directorate of Higher Education, the ministry of National Education, Republic of Indonesia for Program Academic Recharging (PAR-C).

The authors would like thank to Department of Information Science, Saga University for research collaboration. Their support is gratefully acknowledged.

\section{REFERENCES}

[1] Nunzio, G. D, et al. Automatic Lung Segmentation in CT Images with Accurate Handling of Hilar Region. Journal of Digital Imaging. 24, 1, $11-27,2011$

[2] Uppalari, et al. Quantification of pulmonary emphysema from lung computed tomography images. Am. J. Respair. Crit. Care. Med. 156(1), 248-254, 1997.

[3] Chabat, et. al. Obstructive lung diseases: texture classification for differentiation at CT. Radiology 228(3), 871-877, 2003.

[4] Xu, et. al. MDCT-based 3-D texture classification of emphysema and early smoking related lung pathologies, Medical Image Computing and Computer-Assisted Intervention - MICCAI 2007 Lecture Notes in Computer Science, 2007, 4791/2007, 825-833, 2007.

[5] Sluimer, et. al. Computer Aided diagnosis in high resolution CT of the lungs. Med. Phys. 30(12), 3081-3090, 2003.

[6] Sorensen, L., Shaker, S.B., Bruijne, M. L. Texture Classification in the Lung CT using Local Binary Patterns. Medical Image Computing and Computer- Assisted Intervention - MICCAI 2008. LNCS 5241, 925-933, 2008.

[7] Zhao, G and Pietikainen, M. Dynamic Texture Recognition Using Local Binary Patterns with an Application to Facial Expression. IEEE Transaction on Pattern Analysis and Machine Intelligence. 2007.

[8] Gao, X.,Qian, Y., Hui, R., Loomes, M., Comley, R., Barn, B., Chapman, A., and Rix, J. Texture-Based 3D Image Retrieval for Medical Application. IADIS International Conference e-Health 2010,.101-108, 2010.

[9] Arai K, Fundamental theory on wavelet and wavelet analysis, MorikitaShuppan Publishing Co. Ltd., 2000.

[10] Arai K., Self learning for wavelet and wavelet analysis, Kindaikagakusha Publishing Co. Ltd., 2006.

[11] M"aenp“a"a T. and M. Pietik"ainen. In C. Chen and P. Wang, editors, Handbook of Pattern Recognition and Computer Vision, chapter Texture analysis with local bi-nary patterns., pages 197-216. World Scientific, 2005

[12] Specht D.F., Probabilistic Neural Networks for Classification, Mapping, or Associative Memory, IEEE International Conference on Neural Networks, I, 525-532, July 1998

[13] Holder M. and P.O.Lewis, Phylogeny estimation: traditional Baysian approaches, Nature Reviews, 275-284 , 2003

[14] Yee, Paul V. and Haykin, Simon, Regularized Radial Basis Function Networks: Theory and Applications. John Wiley, 2001.

\section{AUTHORS PROFILE}

Kohei Arai received a $\mathrm{PhD}$ from Nihon University in 1982. He was subsequently appointed to the University of Tokyo, CCRS, and the Japan Aerospace Exploration Agency. He was appointed professor at Saga University in 1990. He is also an adjunct professor at the University of Arizona and is Vice Chairman of ICSU/COSPAR Commission A 